\title{
Osteonecrosis of the Knee after Arthroscopic Partial Meniscectomy
}

\author{
Il Jin Son, MD, Min Kyu Kim, MD, Jae Young Kim, MD, and Jin Goo Kim, MD \\ Department of Orthopedic Surgery, Inje University Seoul Paik Hospital, Inje University College of Medicine, Seoul, Korea
}

Osteonecrosis of the femoral condyle is known as an uncommon complication after arthroscopic meniscectomy. The lesion of osteonecrosis can be irreversible, thus early detection of the disease is crucial for treatment. A 50-year-old male patient without known risk factors of osteonecrosis developed increasing knee pain after arthroscopic partial meniscectomy. Magnetic resonance imaging showed rapid progression of osteonecrosis of the medial femoral condyle. Unicompartmental knee arthroplasty was performed after 9 months of conservative therapy. The patient is now free from pain during daily activities. It might be important to remind that if the patient's pain after arthroscopic partial meniscectomy is severe than expected, clinical doctors should pay attention to the possibility of ongoing osteonecrosis of the femoral condyle.

Keywords: Knee, Arthroscopy, Meniscectomy, Osteonecrosis

\section{Introduction}

Osteonecrosis of the knee, first reported by Ahlback et al. ${ }^{1)}$ is characterized by severe pain of sudden onset on the medial side of the knee. The etiology of osteonecrosis has not yet been clearly elucidated, but possible causative factors include sickle cell anemia, Gaucher disease, Caisson disease, corticosteroid treatment, systemic lupus erythematous, and alcohol abuse. Thus, the two major theories of etiology are related to trauma and vascular diseases $^{2}$. The trauma theory implies that repetitive microtrauma in osteoporotic bones results in stress fracture and osteonecrosis. On the other hand, the vascular disease theory explains that an alteration in microcirculation increases bone marrow pressure and microthrombosis disrupts normal blood flow, which leads to the development of osteonecrosis. Osteonecrosis after

Received November 24, 2012; Revised (1st) March 21, 2013;

Accepted June 7, 2013

Correspondence to: Jin Goo Kim, MD

Department of Orthopedic Surgery, Inje University Seoul Paik Hospital, 9 Mareunnae-ro, Jung-gu, Seoul 100-032, Korea

Tel: +82-2-2270-0949, Fax: +82-2-2270-0023

E-mail: boram107@hanmail.net

This is an Open Access article distributed under the terms of the Creative Commons Attribution Non-Commercial License (http://creativecommons.org/licenses/by-nc/3.0/) which permits unrestricted non-commercial use, distribution, and reproduction in any medium, provided the original work is properly cited.

Copyright (C) 2013 KOREAN KNEE SOCIETY arthroscopic meniscectomy is very rare and only about a score of cases has been reported since first described by Brahme et al. ${ }^{3)}$ in 1991. Santori et al. ${ }^{4)}$ suggested that abnormal load transfer after meniscectomy that results in chondral damage, inflammation, edema, and increased bone marrow pressure is the major cause of osteonecrosis. In spite of the rarity, osteonecrosis of the knee after arthroscopic meniscectomy requires early diagnosis and proper treatment to prevent it from developing into a serious complication. In this report, we present a case of osteonecrosis after partial medial meniscectomy that exhibited rapid progression with a review of the literature.

\section{Case Report}

A 50-year-old male visited our clinic with a major complaint of pain in the right knee that had started five days earlier. He had no history of trauma and complained of a pulling sensation behind the right knee and giving way symptoms when walking down stairs. The patient had been working as a medical technician carrying gurneys for 21 years and had no significant medical history or history of trauma, alcohol abuse, and intra-articular steroid injections. Physical examination revealed tenderness over the medial femoral condyle but no edema. The range of motion and lower limb alignment were normal. McMurray's test was negative, joint instability was not present, and early radiography was normal (Fig. 1), Magnetic resonance imaging (MRI) showed

www.jksrr.org 
evidence of posterior meniscal tear without any chondral or bone marrow damage (Fig. 1). Surgical treatment of the posterior tear of the medial meniscus was performed. A complex tear of the posterior horn of the medial meniscus and an International Cartilage Repair Society grade II cartilage lesion (lesions involving $<50 \%$ of the total cartilage thickness) in the medial femoral condyle were observed with arthroscopy (Fig. 2). Surgery was performed under spinal anesthesia while maintaining $70 \mathrm{mmHg}$ of pressure with an arthroscopy pump, and a tourniquet was not used. Partial meniscectomy was performed with a basket forceps and a shaver without using a razor or radiofrequency device and the cartilage damage was treated with debridement. The total surgical time was approximately 20 minutes.

The patient complained of pain at the second postoperative week, which was managed with medication. At the sixth postoperative week, the patient felt severer pain and had the greatest difficulty in walking with straight legs, for which an increased dose of anti-inflammatory analgesics was administered. At the 12th postoperative week, an MRI scan was performed because the pain increased except for temporary relief.
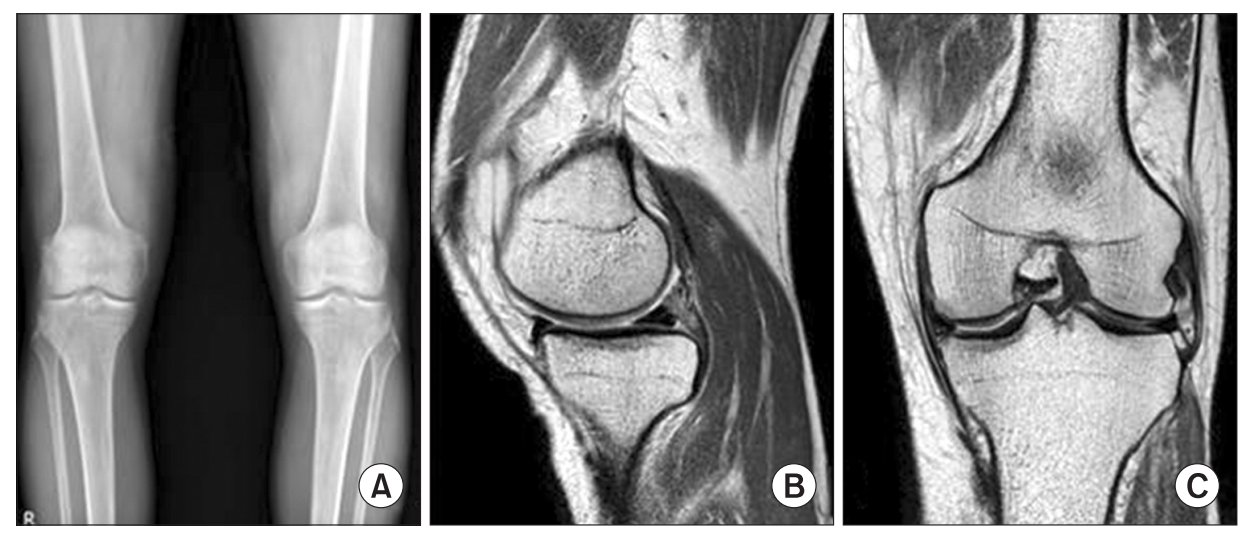

Fig. 1. Preoperative $\mathrm{X}$-ray, magnetic resonance imaging (MRI). (A-C) There was no evidence of osteonecrosis on preoperative radiologic evaluation. (B, C) MRI shows tear of posterior horn of medial meniscus.
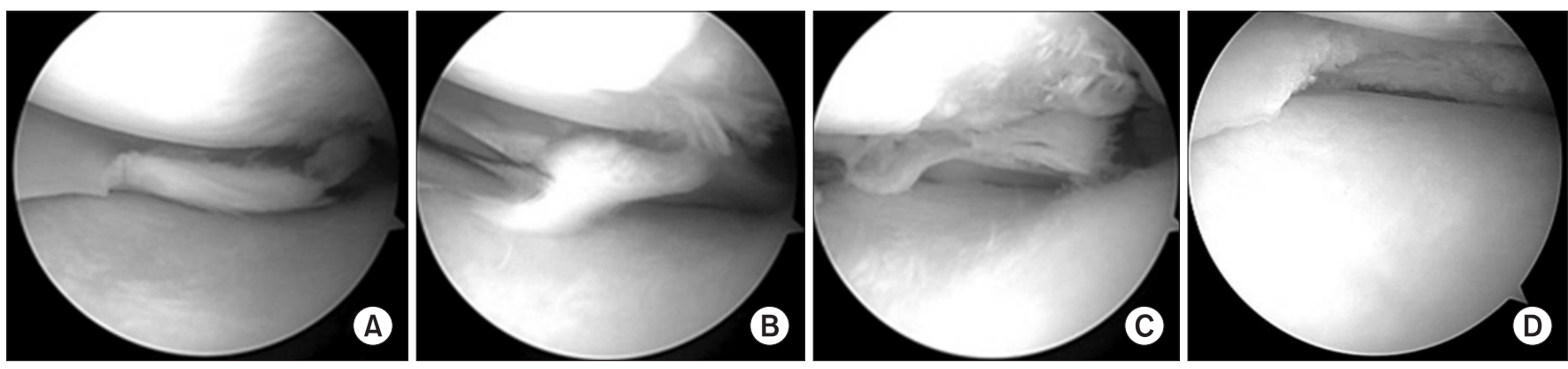

Fig. 2. Intraoperative arthroscopic images. (A, B) A complex tear of posterior horn of the medial meniscus. (C) An International Cartilage Repair Society Grade II cartilage lesion on the medial femoral condyle. (D) Partial meniscectomy was done on the medial meniscus.
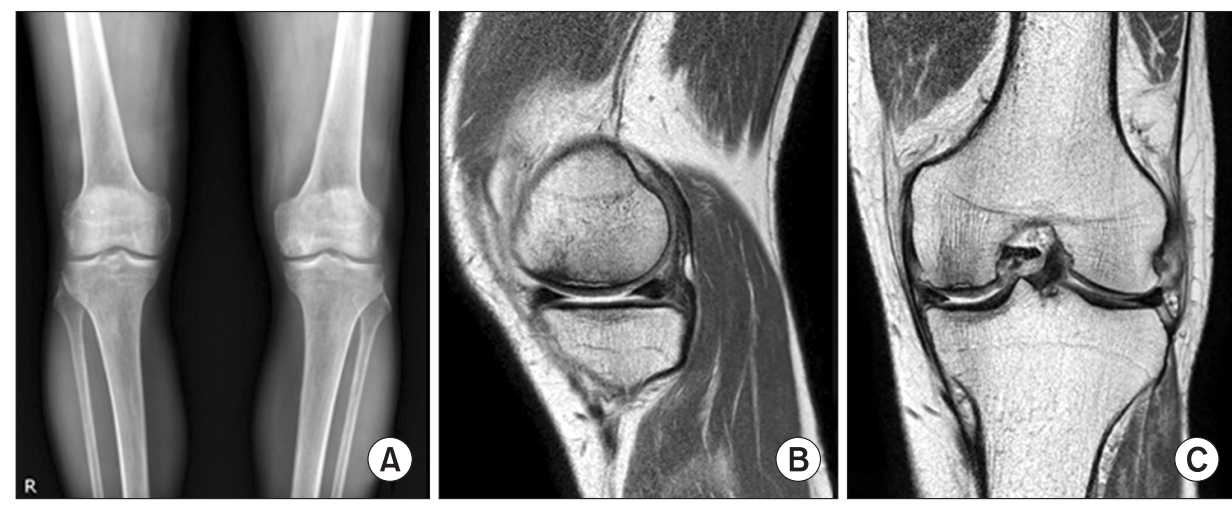

Fig. 3. Radiologic evaluation 3 months after operation. (A) Simple X-ray shows a radiolucent lesion on the medial femoral condyle. (B, C) Coronal \& sagittal modified turbo spin echo images show internal cartilage delamination of the medial femoral condyle. 

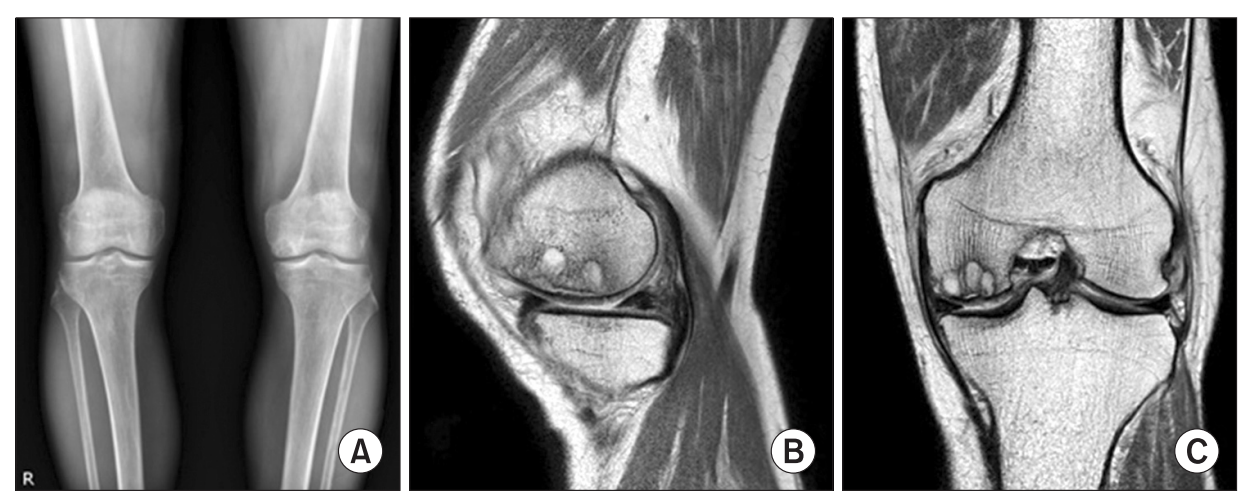

Fig. 4. Radiologic evaluation 9 months after operation. (A) Simple X-ray shows progression of the lesion on the medial femoral condyle. $(\mathrm{B}, \mathrm{C})$ Magnetic resonance images show progressed interval change of delamination of medial femoral condyle and newly developed subchondral cystic change.
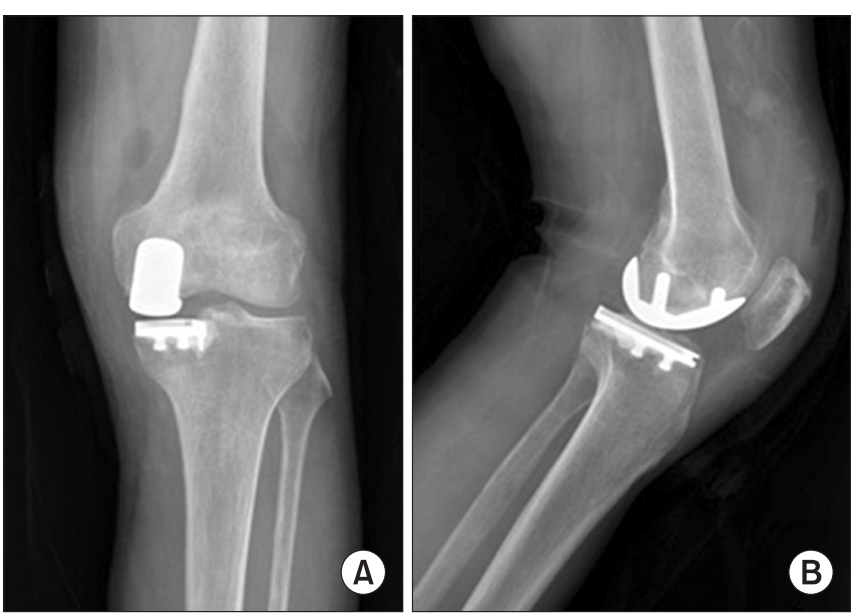

Fig. 5. Radiologic evaluation after unicompartmental knee arthroplasty. (A) Anteroposterior radiograph showing a medial unicondylar knee replacement. (B) Lateral radiograph showing a medial unicondylar knee replacement.

The MRI scan revealed cartilage delamination of the medial femoral condyle that exhibited a low intensity signal on the T1weighted image and a high intensity signal on the T2-weighted image (Fig. 3). Since the symptoms deteriorated during three months of medication therapy, another MRI examination was performed six months postoperatively, which showed more extensive cartilage delamination and cyst formation. An MRI scan taken nine months postoperatively showed an enlarged cyst, increased swelling around the bone, and severe cartilage delamination, all of which were the symptoms of osteonecrosis (Fig. 4). Thus, unicompartmental knee arthroplasty was performed (Fig. 5). Currently, at six months after surgery, the patient has been pain-free and returned to work.

\section{Discussion}

In the present patient who was a 50 -year-old relatively active male without significant medical history, such as arthritis, pain worsened continuously without symptomatic improvement and osteonecrosis progressed rapidly after arthroscopic partial medial meniscectomy that affected approximately $10 \%$ of the total meniscus.

The incidence of osteonecrosis of the femoral condyles after arthroscopic knee surgery has not yet been fully investigated and reported cases are very rare. Moreover, its etiology has not yet been clearly established. Santori et al. ${ }^{4}$ reported two cases of osteonecrosis after meniscectomy that had occurred in a 21-yearold professional football player and a 47-year-old obese woman. They suggested that overloading after meniscectomy results in minor changes in the articular surface that causes impaired circulation in subchondral bone, which eventually leads to the development of osteonecrosis. In the study, most of the symptoms were improved after 45 days of non-weight bearing restriction.

On the other hand, Takeda et al. ${ }^{5)}$ suggested that spontaneous osteonecrosis of the knee is caused by subchondral insufficiency fracture based on the histological findings in 23 patients with the disease: a subchondral fracture and healing reaction were noted in the early stages of the condition and evidence of impaired healing, such as delayed union and nonunion, was observed in the advanced stages. Prues-Latour et al. ${ }^{6}$ encountered 9 cases of osteonecrosis after meniscectomy and suggested that abnormal load transfer after partial meniscectomy causes chondral injury and fracture, which could eventually lead to the development of osteonecrosis. Brahme et al. ${ }^{3)}$ explained the cause of osteonecrosis from a mechanical point of view: more than moderate level of chondral damage was observed in 7 cases of osteonecrosis after meniscectomy and they postulated that the increased intraosseous contact due to chondral damage and meniscectomy could be associated with osteonecrosis.

In a study by Johnson et al..$^{7)}$ medial femoral condyle damage was associated with the poor prognosis of surgery for meniscal tears and chondral lesions, especially for those on the medial 
side, in seven patients who presented with osteonecrosis postoperatively. Akgun et al. ${ }^{8)}$ evaluated five patients with osteonecrosis after arthroscopic meniscal and chondral knee surgery and suggested that development of osteonecrosis should be kept in mind in treating senior osteoarthritic patients with meniscal tears.

The difference between the present case and those in the previous reports is that osteonecrosis progressed rapidly after partial medial meniscectomy that affected only $10 \%$ of the total meniscus in an active male patient without arthritis. We could not clarify whether the meniscectomy was the cause of the osteonecrosis; however, considering that MRI evidence of osteonecrosis was found three months postoperatively in the patient who had had no other findings other than the posterior horn tear, it seems reasonable to assume a causal relationship between abnormal load transfer after meniscectomy and osteonecrosis. On the other hand, it is difficult to rule out the possibility that the patient had a chronic posterior horn meniscus tear and the severe pain that required him to visit the clinic was caused by pre-existing osteonecrosis. If this was the case, osteonecrosis would have progressed regardless of the meniscectomy. Although the preoperative MRI scan did not reveal any lesion on the medial femoral condyle, local tenderness over the medial condyle was more pronounced than the McMurray test result in the physical examination, indicating that osteonecrosis could have developed due to the untreated chronic meniscus tear before meniscectomy.

Pape et al. ${ }^{9)}$ reported that the differential diagnosis of postarthroscopic osteonecrosis of the knee is pre-existing or undiagnosed spontaneous osteonecrosis of the knee that can be determined by bone marrow edema pattern on the pre- and postoperative MRI scans.

In our case, considering the bone marrow edema pattern, the patient's visit might have coincided with the window period of spontaneous osteonecrosis when MRI does not show the lesion in spite of the presence of pain, and the condition deteriorated due to the meniscectomy or irrespective of it. The lesion was located medial to the weight bearing surface on the postoperative MRI and bone marrow edema was not observed in the tibia on the T2-weigted MRI (Fig. 6). Thus, we believe it is difficult to attribute the development of osteonecrosis solely to the abnormal load transfer.

Regarding the treatment of osteonecrosis, the use of non-steroidal anti-inflammatory drugs and analgesics, restriction on weight bearing, and approximately 6 months of conservative treatment should be carried out in the early stages. In the advanced stages, surgical treatment, such as arthroscopic debridement, osteotomy, drilling, or total knee arthoplasty, should be undertaken ${ }^{10)}$. In our patient, symptoms did not improve with the use of non-steroidal anti-inflammatory drugs for a sufficient period of time, followup radiographs showed the lesion was progressive, and the lesion affected the whole medial condyle when the progression stopped. Thus, a replacement surgery was considered necessary, and unicompartmental knee arthoplasty was carried out considering the young age of the patient who had no past medical history and severe deformity.

It is our understanding that the possibility of osteonecrosis or posterior horn root tear should be taken into consideration if pain of sudden onset is abnormally severe compared to the meniscal tear pattern on MRI scan and physical examination findings.

Therefore, if post-meniscectomy symptoms are worse than expectation, thorough examinations including MRI should be conducted to provide proper treatment in case of osteonecrosis of the femoral condyle.

We believe the post-meniscecotmy osteonecrosis case we pre-

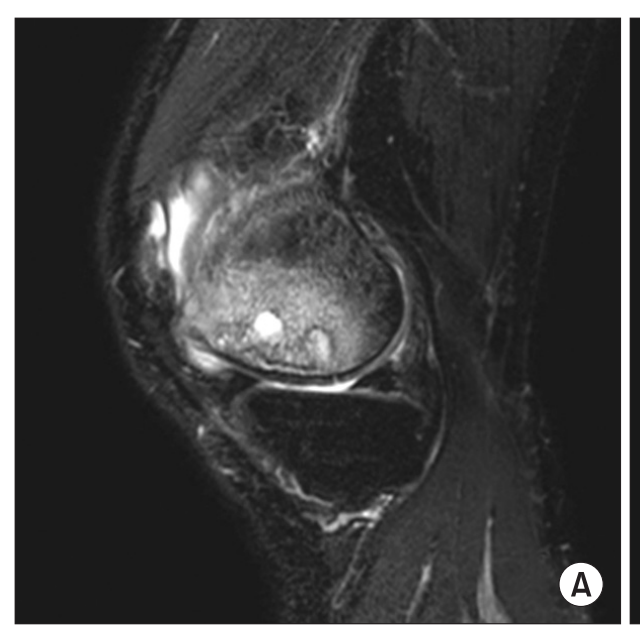

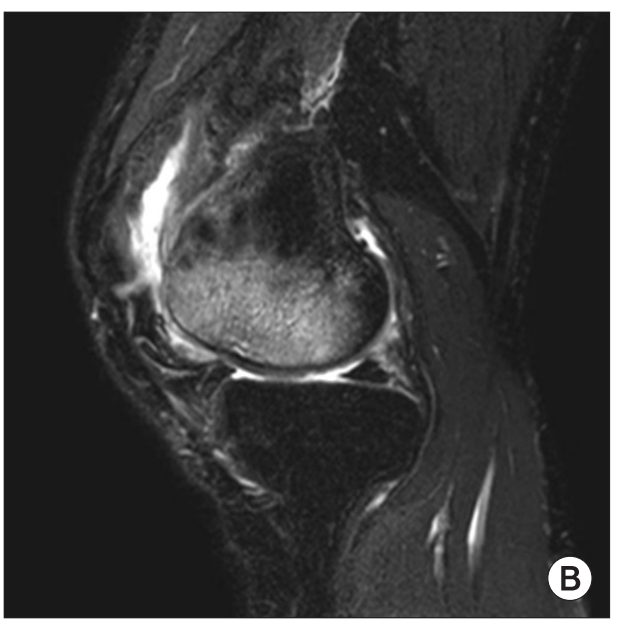

Fig. 6. Radiologic evaluation 9 months after operation. (A, B) T2 sagittal magnetic resonance images show bone marrow edema is only limited to the medial femoral condyle, not involving the tibia plateau. 


\section{Son et al. Knee Osteonecrosis after Arthroscopic Meniscectomy}

sented in this report should be differentiated from the pre-existing spontaneous osteonecrosis. In particular, the osteonecrosis might not have resulted solely from mechanical causes. Therefore, further comprehensive research involving additional cases are considered necessary.

\section{Conflict of Interest}

No potential conflict of interest relevant to this article was reported.

\section{References}

1. Ahlback S, Bauer GC, Bohne WH. Spontaneous osteonecrosis of the knee. Arthritis Rheum. 1968;11:705-33.

2. Aglietti P, Insall JN, Buzzi R, Deschamps G. Idiopathic osteonecrosis of the knee. Aetiology, prognosis and treatment. J Bone Joint Surg Br. 1983;65:588-97.

3. Brahme SK, Fox JM, Ferkel RD, Friedman MJ, Flannigan BD, Resnick DL. Osteonecrosis of the knee after arthroscopic surgery: diagnosis with MR imaging. Radiology. 1991;178:851-3.
4. Santori N, Condello V, Adriani E, Mariani PP. Osteonecrosis after arthroscopic medial meniscectomy. Arthroscopy. 1995;11:220-4.

5. Takeda M, Higuchi H, Kimura M, Kobayashi Y, Terauchi M, Takagishi K. Spontaneous osteonecrosis of the knee: histopathological differences between early and progressive cases. J Bone Joint Surg Br. 2008;90:324-9.

6. Prues-Latour V, Bonvin JC, Fritschy D. Nine cases of osteonecrosis in elderly patients following arthroscopic meniscectomy. Knee Surg Sports Traumatol Arthrosc. 1998;6:142-7.

7. Johnson TC, Evans JA, Gilley JA, DeLee JC. Osteonecrosis of the knee after arthroscopic surgery for meniscal tears and chondral lesions. Arthroscopy. 2000;16:254-61.

8. Akgun RC, Tandogan NR, Karaman A, Akkaya T, Ozgur AF, Tuncay IC. Development of osteonecrosis after arthroscopic meniscal and chondral knee surgery: a report of five cases. Acta Orthop Traumatol Turc. 2007;41:80-8.

9. Pape D, Seil R, Anagnostakos K, Kohn D. Postarthroscopic osteonecrosis of the knee. Arthroscopy. 2007;23:428-38.

10. Lotke PA, Ecker ML. Osteonecrosis of the knee. J Bone Joint Surg Am. 1988;70:470-3. 